\title{
Labelled State Transition Systems
}

\author{
Michał Trybulec \\ YAC Software \\ Warsaw, Poland
}

\begin{abstract}
Summary. This article introduces labelled state transition systems, where transitions may be labelled by words from a given alphabet. Reduction relations from [4] are used to define transitions between states, acceptance of words, and reachable states. Deterministic transition systems are also defined.
\end{abstract}

MML identifier: REWRITE3, version: $7.11 .02 \quad 4.125 .1059$

The articles [1], [8], [2], [11], [6], [17], [7], [9], [16], [15], [14], [4], [10], [13], [3], [12], and [5] provide the notation and terminology for this paper.

\section{Preliminaries}

For simplicity, we adopt the following convention: $x, x_{1}, x_{2}, y, y_{1}, y_{2}, z, z_{1}$, $z_{2}, X, X_{1}, X_{2}$ are sets, $E$ is a non empty set, $e$ is an element of $E, u, v, v_{1}, v_{2}$, $w, w_{1}, w_{2}$ are elements of $E^{\omega}, F, F_{1}, F_{2}$ are subsets of $E^{\omega}$, and $k, l$ are natural numbers.

Next we state a number of propositions:

(1) For every finite sequence $p$ such that $k \in \operatorname{dom} p$ holds $\left(\langle x\rangle^{\frown} p\right)(k+1)=$ $p(k)$.

(2) For every finite sequence $p$ such that $p \neq \emptyset$ there exists a finite sequence $q$ and there exists $x$ such that $p=q^{\frown}\langle x\rangle$ and len $p=\operatorname{len} q+1$.

(3) For every finite sequence $p$ such that $k \in \operatorname{dom} p$ and $k+1 \notin \operatorname{dom} p$ holds len $p=k$.

(4) Let $R$ be a binary relation, $P$ be a reduction sequence w.r.t. $R$, and $q_{1}$, $q_{2}$ be finite sequences. Suppose $P=q_{1} \frown q_{2}$ and len $q_{1}>0$ and len $q_{2}>0$. Then $q_{1}$ is a reduction sequence w.r.t. $R$ and $q_{2}$ is a reduction sequence w.r.t. $R$. 
(5) Let $R$ be a binary relation and $P$ be a reduction sequence w.r.t. $R$. Suppose len $P>1$. Then there exists a reduction sequence $Q$ w.r.t. $R$ such that $\langle P(1)\rangle^{\frown} Q=P$ and len $Q+1=$ len $P$.

(6) Let $R$ be a binary relation and $P$ be a reduction sequence w.r.t. $R$. Suppose len $P>1$. Then there exists a reduction sequence $Q$ w.r.t. $R$ such that $Q^{\frown}\langle P(\operatorname{len} P)\rangle=P$ and $\operatorname{len} Q+1=\operatorname{len} P$.

(7) Let $R$ be a binary relation and $P$ be a reduction sequence w.r.t. $R$. Suppose len $P>1$. Then there exists a reduction sequence $Q$ w.r.t. $R$ such that len $Q+1=\operatorname{len} P$ and for every $k$ such that $k \in \operatorname{dom} Q$ holds $Q(k)=P(k+1)$.

(8) For every binary relation $R$ such that $\langle x, y\rangle$ is a reduction sequence w.r.t. $R$ holds $\langle x, y\rangle \in R$.

(9) If $w=u^{\frown} v$, then len $u \leq$ len $w$ and len $v \leq$ len $w$.

(10) If $w=u^{\frown} v$ and $u \neq\langle\rangle_{E}$ and $v \neq\langle\rangle_{E}$, then len $u<\operatorname{len} w$ and len $v<$ len $w$.

(11) If $w_{1}{ }^{\wedge} v_{1}=w_{2} \frown v_{2}$ and if len $w_{1}=$ len $w_{2}$ or len $v_{1}=$ len $v_{2}$, then $w_{1}=w_{2}$ and $v_{1}=v_{2}$.

(12) If $w_{1} \frown v_{1}=w_{2} \frown v_{2}$ and if len $w_{1} \leq$ len $w_{2}$ or len $v_{1} \geq$ len $v_{2}$, then there exists $u$ such that $w_{1} \frown u=w_{2}$ and $v_{1}=u^{\frown} v_{2}$.

(13) If $w_{1} \frown v_{1}=w_{2} \frown v_{2}$, then there exists $u$ such that $w_{1} \frown u=w_{2}$ and $v_{1}=u^{\curvearrowleft} v_{2}$ or there exists $u$ such that $w_{2}{ }^{\frown} u=w_{1}$ and $v_{2}=u^{\curvearrowleft} v_{1}$.

Let us consider $X$. We introduce transition-systems over $X$ which are extensions of 1-sorted structure and are systems

$\langle$ a carrier, a transition 〉,

where the carrier is a set and the transition is a relation between the carrier $\times$ $X$ and the carrier.

\section{Transition Systems over Subsets of $E^{\omega}$}

Let us consider $E, F$ and let $\mathfrak{T}$ be a transition-system over $F$. We say that $\mathfrak{T}$ is deterministic if and only if the conditions (Def. 1) are satisfied.

(Def. 1)(i) The transition of $\mathfrak{T}$ is a function,

(ii) \langle\rangle$_{E} \notin \operatorname{rng} \operatorname{dom}($ the transition of $\mathfrak{T}$ ), and

(iii) for every element $s$ of $\mathfrak{T}$ and for all $u, v$ such that $u \neq v$ and $\langle s$, $u\rangle \in \operatorname{dom}$ (the transition of $\mathfrak{T}$ ) and $\langle s, v\rangle \in \operatorname{dom}$ (the transition of $\mathfrak{T}$ ) it is not true that there exists $w$ such that $u^{\frown} w=v$ or $v^{\frown} w=u$.

We now state the proposition

(14) For every transition-system $\mathfrak{T}$ over $F$ such that dom (the transition of $\mathfrak{T})=\emptyset$ holds $\mathfrak{T}$ is deterministic. 
Let us consider $E, F$. Observe that there exists a transition-system over $F$ which is strict, non empty, finite, and deterministic.

\section{Productions}

Let us consider $X$, let $\mathfrak{T}$ be a transition-system over $X$, and let us consider $x, y, z$. The predicate $x, y \rightarrow \mathfrak{T} z$ is defined by:

(Def. 2) $\langle\langle x, y\rangle, z\rangle \in$ the transition of $\mathfrak{T}$.

We now state several propositions:

(15) Let $\mathfrak{T}$ be a transition-system over $X$. Suppose $x, y \rightarrow \mathfrak{T} z$. Then

(i) $\quad x \in \mathfrak{T}$,

(ii) $y \in X$,

(iii) $z \in \mathfrak{T}$,

(iv) $\quad x \in \operatorname{dom} \operatorname{dom}($ the transition of $\mathfrak{T})$,

(v) $y \in \operatorname{rng} \operatorname{dom}($ the transition of $\mathfrak{T}$ ), and

(vi) $z \in \operatorname{rng}$ (the transition of $\mathfrak{T}$ ).

(16) Let $\mathfrak{T}_{1}$ be a transition-system over $X_{1}$ and $\mathfrak{T}_{2}$ be a transition-system over $X_{2}$. Suppose the transition of $\mathfrak{T}_{1}=$ the transition of $\mathfrak{T}_{2}$. If $x, y \rightarrow \mathfrak{T}_{1} z$, then $x, y \rightarrow \mathfrak{T}_{2} z$.

(17) Let $\mathfrak{T}$ be a transition-system over $F$. Suppose the transition of $\mathfrak{T}$ is a function. If $x, y \rightarrow \mathfrak{T} z_{1}$ and $x, y \rightarrow \mathfrak{T} z_{2}$, then $z_{1}=z_{2}$.

(18) For every deterministic transition-system $\mathfrak{T}$ over $F$ such that \langle\rangle$_{E} \notin$ rng dom (the transition of $\mathfrak{T}$ ) holds $x,\langle\rangle_{E} \not \rightarrow \mathfrak{T} y$.

(19) Let $\mathfrak{T}$ be a deterministic transition-system over $F$. If $u \neq v$ and $x, u \rightarrow \mathfrak{T}$ $z_{1}$ and $x, v \rightarrow \mathfrak{T} z_{2}$, then it is not true that there exists $w$ such that $u^{\frown} w=v$ or $v^{\frown} w=u$.

\section{Direct Transitions}

Let us consider $E, F$, let $\mathfrak{T}$ be a transition-system over $F$, and let us consider $x_{1}, x_{2}, y_{1}, y_{2}$. The predicate $x_{1}, x_{2} \Rightarrow \mathfrak{T} y_{1}, y_{2}$ is defined as follows:

(Def. 3) There exist $v, w$ such that $v=y_{2}$ and $x_{1}, w \rightarrow \mathfrak{T} y_{1}$ and $x_{2}=w^{\frown} v$.

The following propositions are true:

(20) Let $\mathfrak{T}$ be a transition-system over $F$. Suppose $x_{1}, x_{2} \Rightarrow \mathfrak{T} y_{1}, y_{2}$. Then $x_{1}, y_{1} \in \mathfrak{T}$ and $x_{2}, y_{2} \in E^{\omega}$ and $x_{1} \in \operatorname{dom} \operatorname{dom}$ (the transition of $\mathfrak{T}$ ) and $y_{1} \in \operatorname{rng}$ (the transition of $\mathfrak{T}$ ).

(21) Let $\mathfrak{T}_{1}$ be a transition-system over $F_{1}$ and $\mathfrak{T}_{2}$ be a transition-system over $F_{2}$. Suppose the transition of $\mathfrak{T}_{1}=$ the transition of $\mathfrak{T}_{2}$ and $x_{1}, x_{2} \Rightarrow \mathfrak{T}_{1}$ $y_{1}, y_{2}$. Then $x_{1}, x_{2} \Rightarrow \mathfrak{T}_{2} y_{1}, y_{2}$. 
(22) For every transition-system $\mathfrak{T}$ over $F$ such that $x, u \Rightarrow \mathfrak{T} y, v$ there exists $w$ such that $x, w \rightarrow \mathfrak{T} y$ and $u=w^{\frown} v$.

(23) For every transition-system $\mathfrak{T}$ over $F$ holds $x, y \rightarrow \mathfrak{T} z$ iff $x, y \Rightarrow \mathfrak{T} z,\langle\rangle_{E}$.

(24) For every transition-system $\mathfrak{T}$ over $F$ holds $x, v \rightarrow \mathfrak{T} y$ iff $x, v^{\frown} w \Rightarrow \mathfrak{T} y, w$.

(25) For every transition-system $\mathfrak{T}$ over $F$ such that $x, u \Rightarrow \mathfrak{T} y, v$ holds $x, u^{\frown}$ $w \Rightarrow \mathfrak{T} y, v^{\frown} w$.

(26) For every transition-system $\mathfrak{T}$ over $F$ such that $x, u \Rightarrow \mathfrak{T} y, v$ holds len $u \geq$ len $v$.

(27) Let $\mathfrak{T}$ be a transition-system over $F$. Suppose the transition of $\mathfrak{T}$ is a function. If $x_{1}, x_{2} \Rightarrow \mathfrak{T} y_{1}, z$ and $x_{1}, x_{2} \Rightarrow \mathfrak{T} y_{2}, z$, then $y_{1}=y_{2}$.

(28) For every transition-system $\mathfrak{T}$ over $F$ such that \langle\rangle$_{E} \notin$ rng dom (the transition of $\mathfrak{T}$ ) holds $x, z \neq \neq_{\mathfrak{T}} y, z$.

(29) For every transition-system $\mathfrak{T}$ over $F$ such that \langle\rangle$_{E} \notin$ rng dom (the transition of $\mathfrak{T}$ ) holds if $x, u \Rightarrow \mathfrak{T} y, v$, then len $u>\operatorname{len} v$.

(30) For every deterministic transition-system $\mathfrak{T}$ over $F$ such that $x_{1}, x_{2} \Rightarrow \mathfrak{T}$ $y_{1}, z_{1}$ and $x_{1}, x_{2} \Rightarrow \mathfrak{T} y_{2}, z_{2}$ holds $y_{1}=y_{2}$ and $z_{1}=z_{2}$.

\section{ReduCtion RELATiON}

In the sequel $\mathfrak{T}$ is a non empty transition-system over $F, s, t$ are elements of $\mathfrak{T}$, and $S$ is a subset of $\mathfrak{T}$.

Let us consider $E, F, \mathfrak{T}$. The functor $\Rightarrow \mathfrak{T}$ yielding a binary relation on (the carrier of $\mathfrak{T}) \times E^{\omega}$ is defined as follows:

(Def. 4) $\left\langle\left\langle x_{1}, x_{2}\right\rangle,\left\langle y_{1}, y_{2}\right\rangle\right\rangle \in \Rightarrow \mathfrak{T}$ iff $x_{1}, x_{2} \Rightarrow \mathfrak{T} y_{1}, y_{2}$.

The following propositions are true:

(31) If $\langle x, y\rangle \in \Rightarrow_{\mathfrak{T}}$, then there exist $s, v, t, w$ such that $x=\langle s, v\rangle$ and $y=\langle t, w\rangle$.

(32) Suppose $\left\langle\left\langle x_{1}, x_{2}\right\rangle,\left\langle y_{1}, y_{2}\right\rangle\right\rangle \in \Rightarrow \mathfrak{T}$. Then $x_{1}, y_{1} \in \mathfrak{T}$ and $x_{2}, y_{2} \in E^{\omega}$ and $x_{1} \in \operatorname{dom} \operatorname{dom}\left(\right.$ the transition of $\mathfrak{T}$ ) and $y_{1} \in \operatorname{rng}$ (the transition of $\mathfrak{T}$ ).

(33) If $x \in \Rightarrow_{\mathfrak{T}}$, then there exist $s, t, v, w$ such that $x=\langle\langle s, v\rangle,\langle t, w\rangle\rangle$.

(34) Let $\mathfrak{T}_{1}$ be a non empty transition-system over $F_{1}$ and $\mathfrak{T}_{2}$ be a non empty transition-system over $F_{2}$. Suppose the carrier of $\mathfrak{T}_{1}=$ the carrier of $\mathfrak{T}_{2}$ and the transition of $\mathfrak{T}_{1}=$ the transition of $\mathfrak{T}_{2}$. Then $\Rightarrow \mathfrak{T}_{1}=\Rightarrow_{\mathfrak{T}_{2}}$.

(35) If $\left\langle\left\langle x_{1}, x_{2}\right\rangle,\left\langle y_{1}, y_{2}\right\rangle\right\rangle \in \Rightarrow \mathfrak{T}$, then there exist $v, w$ such that $v=y_{2}$ and $x_{1}, w \rightarrow \mathfrak{T} y_{1}$ and $x_{2}=w^{\frown} v$.

(36) If $\langle\langle x, u\rangle,\langle y, v\rangle\rangle \in \Rightarrow \mathfrak{T}$, then there exists $w$ such that $x, w \rightarrow \mathfrak{T} y$ and $u=w^{\frown} v$.

(37) $\quad x, y \rightarrow \mathfrak{T} z$ iff $\left\langle\langle x, y\rangle,\left\langle z,\langle\rangle_{E}\right\rangle\right\rangle \in \Rightarrow \mathfrak{T}$.

(38) $x, v \rightarrow \mathfrak{T} y$ iff $\left\langle\left\langle x, v^{\frown} w\right\rangle,\langle y, w\rangle\right\rangle \in \Rightarrow \mathfrak{T}$. 
(39) If $\langle\langle x, u\rangle,\langle y, v\rangle\rangle \in \Rightarrow_{\mathfrak{T}}$, then $\left\langle\left\langle x, u^{\frown} w\right\rangle,\left\langle y, v^{\frown} w\right\rangle\right\rangle \in \Rightarrow_{\mathfrak{T}}$.

(40) If $\langle\langle x, u\rangle,\langle y, v\rangle\rangle \in \Rightarrow \mathfrak{T}$, then len $u \geq \operatorname{len} v$.

(41) If the transition of $\mathfrak{T}$ is a function, then if $\left\langle x,\left\langle y_{1}, z\right\rangle\right\rangle,\left\langle x,\left\langle y_{2}, z\right\rangle\right\rangle \in \Rightarrow \mathfrak{T}$, then $y_{1}=y_{2}$.

(42) If \langle\rangle$_{E} \notin \operatorname{rng} \operatorname{dom}($ the transition of $\mathfrak{T}$ ), then if $\langle\langle x, u\rangle,\langle y, v\rangle\rangle \in \Rightarrow \mathfrak{T}$, then len $u>$ len $v$.

(43) If \langle\rangle$_{E} \notin \operatorname{rng} \operatorname{dom}$ (the transition of $\mathfrak{T}$ ), then $\langle\langle x, z\rangle,\langle y, z\rangle\rangle \notin \Rightarrow \mathfrak{T}$.

(44) If $\mathfrak{T}$ is deterministic, then if $\left\langle x, y_{1}\right\rangle,\left\langle x, y_{2}\right\rangle \in \Rightarrow \mathfrak{T}$, then $y_{1}=y_{2}$.

(45) If $\mathfrak{T}$ is deterministic, then if $\left\langle x,\left\langle y_{1}, z_{1}\right\rangle\right\rangle,\left\langle x,\left\langle y_{2}, z_{2}\right\rangle\right\rangle \in \Rightarrow_{\mathfrak{T}}$, then $y_{1}=$ $y_{2}$ and $z_{1}=z_{2}$.

(46) If $\mathfrak{T}$ is deterministic, then $\Rightarrow_{\mathfrak{T}}$ is function-like.

\section{Reduction Sequences}

Let us consider $x, E$. The functor $\operatorname{dim}_{2}(x, E)$ yields an element of $E^{\omega}$ and is defined as follows:

(Def. 5) $\operatorname{dim}_{2}(x, E)=\left\{\begin{array}{l}x_{2}, \text { if there exist } y, u \text { such that } x=\langle y, u\rangle, \\ \emptyset, \text { otherwise. }\end{array}\right.$

Next we state a number of propositions:

(47) Let $P$ be a reduction sequence w.r.t. $\Rightarrow \mathfrak{T}$ and given $k$. If $k, k+1 \in \operatorname{dom} P$, then there exist $s, v, t, w$ such that $P(k)=\langle s, v\rangle$ and $P(k+1)=\langle t, w\rangle$.

(48) Let $P$ be a reduction sequence w.r.t. $\Rightarrow \mathfrak{T}$ and given $k$. If $k, k+1 \in \operatorname{dom} P$, then $P(k)=\left\langle P(k)_{\mathbf{1}}, P(k)_{\mathbf{2}}\right\rangle$ and $P(k+1)=\left\langle P(k+1)_{\mathbf{1}}, P(k+1)_{\mathbf{2}}\right\rangle$.

(49) Let $P$ be a reduction sequence w.r.t. $\Rightarrow \mathfrak{T}$ and given $k$. Suppose $k, k+1 \in$ $\operatorname{dom} P$. Then

(i) $P(k)_{\mathbf{1}} \in \mathfrak{T}$,

(ii) $P(k)_{\mathbf{2}} \in E^{\omega}$,

(iii) $P(k+1)_{1} \in \mathfrak{T}$,

(iv) $P(k+1)_{\mathbf{2}} \in E^{\omega}$,

(v) $P(k)_{1} \in \operatorname{dom} \operatorname{dom}$ (the transition of $\mathfrak{T}$ ), and

(vi) $P(k+1)_{1} \in \operatorname{rng}$ (the transition of $\left.\mathfrak{T}\right)$.

(50) Let $\mathfrak{T}_{1}$ be a non empty transition-system over $F_{1}$ and $\mathfrak{T}_{2}$ be a non empty transition-system over $F_{2}$. Suppose the carrier of $\mathfrak{T}_{1}=$ the carrier of $\mathfrak{T}_{2}$ and the transition of $\mathfrak{T}_{1}=$ the transition of $\mathfrak{T}_{2}$. Then every reduction sequence w.r.t. $\Rightarrow \mathfrak{T}_{1}$ is a reduction sequence w.r.t. $\Rightarrow \mathfrak{T}_{2}$.

(51) Let $P$ be a reduction sequence w.r.t. $\Rightarrow \mathfrak{T}$. If there exist $x, u$ such that $P(1)=\langle x, u\rangle$, then for every $k$ such that $k \in \operatorname{dom} P$ holds $\operatorname{dim}_{2}(P(k), E)=P(k)_{2}$.

(52) Let $P$ be a reduction sequence w.r.t. $\Rightarrow \mathfrak{T}$. If $P($ len $P)=\langle y, w\rangle$, then for every $k$ such that $k \in \operatorname{dom} P$ there exists $u$ such that $P(k)_{\mathbf{2}}=u^{\frown} w$. 
(53) For every reduction sequence $P$ w.r.t. $\Rightarrow \mathfrak{T}$ such that $P(1)=\langle x, v\rangle$ and $P($ len $P)=\langle y, w\rangle$ there exists $u$ such that $v=u^{\frown} w$.

(54) Let $P$ be a reduction sequence w.r.t. $\Rightarrow \mathfrak{T}$. If $P(1)=\langle x, u\rangle$ and $P(\operatorname{len} P)=\langle y, u\rangle$, then for every $k$ such that $k \in \operatorname{dom} P$ holds $P(k)_{\mathbf{2}}=u$.

(55) Let $P$ be a reduction sequence w.r.t. $\Rightarrow \mathfrak{T}$ and given $k$. Suppose $k, k+1 \in$ $\operatorname{dom} P$. Then there exist $v, w$ such that $v=P(k+1)_{\mathbf{2}}$ and $P(k)_{\mathbf{1}}, w \rightarrow \mathfrak{T}$ $P(k+1)_{1}$ and $P(k)_{2}=w^{\frown} v$.

(56) Let $P$ be a reduction sequence w.r.t. $\Rightarrow \mathfrak{T}$ and given $k$. Suppose $k, k+1 \in$ $\operatorname{dom} P$ and $P(k)=\langle x, u\rangle$ and $P(k+1)=\langle y, v\rangle$. Then there exists $w$ such that $x, w \rightarrow \mathfrak{T} y$ and $u=w^{\frown} v$.

(57) $\quad x, y \rightarrow \mathfrak{T} z$ iff $\left\langle\langle x, y\rangle,\left\langle z,\langle\rangle_{E}\right\rangle\right\rangle$ is a reduction sequence w.r.t. $\Rightarrow \mathfrak{T}$.

(58) $\quad x, v \rightarrow \mathfrak{T} y$ iff $\left\langle\left\langle x, v^{\frown} w\right\rangle,\langle y, w\rangle\right\rangle$ is a reduction sequence w.r.t. $\Rightarrow \mathfrak{T}$.

(59) For every reduction sequence $P$ w.r.t. $\Rightarrow \mathfrak{T}$ such that $P(1)=\langle x, v\rangle$ and $P(\operatorname{len} P)=\langle y, w\rangle$ holds len $v \geq$ len $w$.

(60) Suppose \langle\rangle$_{E} \notin$ rng dom (the transition of $\mathfrak{T}$ ). Let $P$ be a reduction sequence w.r.t. $\Rightarrow \mathfrak{T}$. If $P(1)=\langle x, u\rangle$ and $P(\operatorname{len} P)=\langle y, u\rangle$, then len $P=1$ and $x=y$.

(61) Suppose \langle\rangle$_{E} \notin$ rng dom (the transition of $\left.\mathfrak{T}\right)$. Let $P$ be a reduction sequence w.r.t. $\Rightarrow \mathfrak{T}$. If $P(1)_{\mathbf{2}}=P(\operatorname{len} P)_{\mathbf{2}}$, then len $P=1$.

(62) Suppose \langle\rangle$_{E} \notin \operatorname{rng} \operatorname{dom}($ the transition of $\mathfrak{T}$ ). Let $P$ be a reduction sequence w.r.t. $\Rightarrow \mathfrak{T}$. If $P(1)=\langle x, u\rangle$ and $P(\operatorname{len} P)=\left\langle y,\langle\rangle_{E}\right\rangle$, then len $P \leq$ len $u+1$.

(63) Suppose \langle\rangle$_{E} \notin \operatorname{rng} \operatorname{dom}$ (the transition of $\mathfrak{T}$ ). Let $P$ be a reduction sequence w.r.t. $\Rightarrow \mathfrak{T}$. If $P(1)=\langle x,\langle e\rangle\rangle$ and $P(\operatorname{len} P)=\left\langle y,\langle\rangle_{E}\right\rangle$, then len $P=2$.

(64) Suppose \langle\rangle$_{E} \notin \operatorname{rng}$ dom (the transition of $\mathfrak{T}$ ). Let $P$ be a reduction sequence w.r.t. $\Rightarrow \mathfrak{T}$. If $P(1)=\langle x, v\rangle$ and $P(\operatorname{len} P)=\langle y, w\rangle$, then len $v>$ len $w$ or len $P=1$ and $x=y$ and $v=w$.

(65) Suppose \langle\rangle$_{E} \notin$ rng dom (the transition of $\mathfrak{T}$ ). Let $P$ be a reduction sequence w.r.t. $\Rightarrow_{\mathfrak{T}}$ and given $k$. If $k, k+1 \in \operatorname{dom} P$, then $P(k)_{\mathbf{2}} \neq P(k+1)_{\mathbf{2}}$.

(66) Suppose \langle\rangle$_{E} \notin \mathrm{rng} \operatorname{dom}$ (the transition of $\mathfrak{T}$ ). Let $P$ be a reduction sequence w.r.t. $\Rightarrow \mathfrak{T}$ and given $k, l$. If $k, l \in \operatorname{dom} P$ and $k<l$, then $P(k)_{\mathbf{2}} \neq P(l)_{\mathbf{2}}$.

(67) Suppose $\mathfrak{T}$ is deterministic. Let $P, Q$ be reduction sequences w.r.t. $\Rightarrow \mathfrak{T}$. If $P(1)=Q(1)$, then for every $k$ such that $k \in \operatorname{dom} P$ and $k \in \operatorname{dom} Q$ holds $P(k)=Q(k)$.

(68) If $\mathfrak{T}$ is deterministic, then for all reduction sequences $P, Q$ w.r.t. $\Rightarrow \mathfrak{T}$ such that $P(1)=Q(1)$ and len $P=\operatorname{len} Q$ holds $P=Q$.

(69) Suppose $\mathfrak{T}$ is deterministic. Let $P, Q$ be reduction sequences w.r.t. $\Rightarrow \mathfrak{T}$. If $P(1)=Q(1)$ and $P(\operatorname{len} P)_{\mathbf{2}}=Q(\operatorname{len} Q)_{\mathbf{2}}$, then $P=Q$. 


\section{REduCtions}

The following propositions are true:

(70) If $\Rightarrow \mathfrak{T}$ reduces $\langle x, v\rangle$ to $\langle y, w\rangle$, then there exists $u$ such that $v=u^{\frown} w$.

(71) If $\Rightarrow \mathfrak{T}$ reduces $\langle x, u\rangle$ to $\langle y, v\rangle$, then $\Rightarrow_{\mathfrak{T}}$ reduces $\left\langle x, u^{\frown} w\right\rangle$ to $\left\langle y, v^{\frown} w\right\rangle$.

(72) If $x, y \rightarrow \mathfrak{T} z$, then $\Rightarrow \mathfrak{T}$ reduces $\langle x, y\rangle$ to $\left\langle z,\langle\rangle_{E}\right\rangle$.

(73) If $x, v \rightarrow \mathfrak{T} y$, then $\Rightarrow \mathfrak{T}$ reduces $\left\langle x, v^{\frown} w\right\rangle$ to $\langle y, w\rangle$.

(74) If $x_{1}, x_{2} \Rightarrow \mathfrak{T} y_{1}, y_{2}$, then $\Rightarrow \mathfrak{T}$ reduces $\left\langle x_{1}, x_{2}\right\rangle$ to $\left\langle y_{1}, y_{2}\right\rangle$.

(75) If $\Rightarrow \mathfrak{T}$ reduces $\langle x, v\rangle$ to $\langle y, w\rangle$, then len $v \geq \operatorname{len} w$.

(76) If $\Rightarrow \mathfrak{T}$ reduces $\langle x, w\rangle$ to $\left\langle y, v^{\frown} w\right\rangle$, then $v=\langle\rangle_{E}$.

(77) If \langle\rangle$_{E} \notin \operatorname{rng} \operatorname{dom}($ the transition of $\mathfrak{T}$ ), then if $\Rightarrow \mathfrak{T}$ reduces $\langle x, v\rangle$ to $\langle y$, $w\rangle$, then len $v>$ len $w$ or $x=y$ and $v=w$.

(78) If \langle\rangle$_{E} \notin \operatorname{rng}$ dom (the transition of $\mathfrak{T}$ ), then if $\Rightarrow \mathfrak{T}$ reduces $\langle x, u\rangle$ to $\langle y$, $u\rangle$, then $x=y$.

(79) If \langle\rangle$_{E} \notin \operatorname{rng} \operatorname{dom}$ (the transition of $\mathfrak{T}$ ), then if $\Rightarrow \mathfrak{T}$ reduces $\langle x,\langle e\rangle\rangle$ to $\langle y$, \langle\rangle$\left._{E}\right\rangle$, then $\left\langle\langle x,\langle e\rangle\rangle,\left\langle y,\langle\rangle_{E}\right\rangle\right\rangle \in \Rightarrow \mathfrak{T}$.

(80) If $\mathfrak{T}$ is deterministic, then if $\Rightarrow \mathfrak{T}$ reduces $x$ to $\left\langle y_{1}, z\right\rangle$ and $\Rightarrow_{\mathfrak{T}}$ reduces $x$ to $\left\langle y_{2}, z\right\rangle$, then $y_{1}=y_{2}$.

\section{Transitions}

Let us consider $E, F, \mathfrak{T}, x_{1}, x_{2}, y_{1}, y_{2}$. The predicate $x_{1}, x_{2} \Rightarrow_{\mathfrak{T}}^{*} y_{1}, y_{2}$ is defined as follows:

(Def. 6) $\Rightarrow$ T reduces $\left\langle x_{1}, x_{2}\right\rangle$ to $\left\langle y_{1}, y_{2}\right\rangle$.

We now state a number of propositions:

(81) Let $\mathfrak{T}_{1}$ be a non empty transition-system over $F_{1}$ and $\mathfrak{T}_{2}$ be a non empty transition-system over $F_{2}$. Suppose the carrier of $\mathfrak{T}_{1}=$ the carrier of $\mathfrak{T}_{2}$ and the transition of $\mathfrak{T}_{1}=$ the transition of $\mathfrak{T}_{2}$. If $x_{1}, x_{2} \Rightarrow{ }_{\mathfrak{T}_{1}}^{*} y_{1}, y_{2}$, then $x_{1}, x_{2} \Rightarrow_{\mathfrak{T}_{2}}^{*} y_{1}, y_{2}$.

(82) $x, y \Rightarrow{ }_{\mathfrak{T}}^{*} x, y$.

(83) If $x_{1}, x_{2} \Rightarrow_{\mathfrak{T}}^{*} y_{1}, y_{2}$ and $y_{1}, y_{2} \Rightarrow_{\mathfrak{T}}^{*} z_{1}, z_{2}$, then $x_{1}, x_{2} \Rightarrow_{\mathfrak{T}}^{*} z_{1}, z_{2}$.

(84) If $x, y \rightarrow \mathfrak{T} z$, then $x, y \Rightarrow_{\mathfrak{T}}^{*} z,\langle\rangle_{E}$.

(85) If $x, v \rightarrow \mathfrak{T} y$, then $x, v^{\frown} w \Rightarrow_{\mathfrak{T}}^{*} y, w$.

(86) If $x, u \Rightarrow_{\mathfrak{T}}^{*} y, v$, then $x, u^{\frown} w \Rightarrow_{\mathfrak{T}}^{*} y, v^{\frown} w$.

(87) If $x_{1}, x_{2} \Rightarrow \mathfrak{T} y_{1}, y_{2}$, then $x_{1}, x_{2} \Rightarrow_{\mathfrak{T}}^{*} y_{1}, y_{2}$.

(88) If $x, v \Rightarrow_{\mathfrak{T}}^{*} y, w$, then there exists $u$ such that $v=u^{\frown} w$.

(89) If $x, v \Rightarrow_{\mathfrak{T}}^{*} y, w$, then len $w \leq \operatorname{len} v$.

(90) If $x, w \Rightarrow_{\mathfrak{T}}^{*} y, v^{\frown} w$, then $v=\langle\rangle_{E}$. 
(91) If \langle\rangle$_{E} \notin \operatorname{rng} \operatorname{dom}\left(\right.$ the transition of $\mathfrak{T}$ ), then $x, u \Rightarrow_{\mathfrak{T}}^{*} y, u$ iff $x=y$.

(92) If \langle\rangle$_{E} \notin \operatorname{rng} \operatorname{dom}\left(\right.$ the transition of $\mathfrak{T}$ ), then if $x,\langle e\rangle \Rightarrow_{\mathfrak{T}}^{*} y,\langle\rangle_{E}$, then $x,\langle e\rangle \Rightarrow \mathfrak{T} y,\langle\rangle_{E}$.

(93) If $\mathfrak{T}$ is deterministic, then if $x_{1}, x_{2} \Rightarrow_{\mathfrak{T}}^{*} y_{1}, z$ and $x_{1}, x_{2} \Rightarrow_{\mathfrak{T}}^{*} y_{2}, z$, then $y_{1}=y_{2}$.

\section{Acceptance of Words}

Let us consider $E, F, \mathfrak{T}, x_{1}, x_{2}, y$. The predicate $x_{1}, x_{2} \Rightarrow_{\mathfrak{T}}^{*} y$ is defined as follows:

(Def. 7) $x_{1}, x_{2} \Rightarrow_{\mathfrak{T}}^{*} y,\langle\rangle_{E}$.

We now state several propositions:

(94) Let $\mathfrak{T}_{1}$ be a non empty transition-system over $F_{1}$ and $\mathfrak{T}_{2}$ be a non empty transition-system over $F_{2}$. Suppose the carrier of $\mathfrak{T}_{1}=$ the carrier of $\mathfrak{T}_{2}$ and the transition of $\mathfrak{T}_{1}=$ the transition of $\mathfrak{T}_{2}$. If $x, y \Rightarrow_{\mathfrak{T}_{1}}^{*} z$, then $x, y \Rightarrow_{\mathfrak{T}_{2}}^{*} z$.

(95) $x,\langle\rangle_{E} \Rightarrow_{\mathfrak{T}}^{*} x$.

(96) If $x, u \Rightarrow_{\mathfrak{T}}^{*} y$, then $x, u^{\frown} v \Rightarrow_{\mathfrak{T}}^{*} y, v$.

(97) If $x, y \rightarrow \mathfrak{T} z$, then $x, y \Rightarrow_{\mathfrak{T}}^{*} z$.

(98) If $x_{1}, x_{2} \Rightarrow \mathfrak{T} y,\langle\rangle_{E}$, then $x_{1}, x_{2} \Rightarrow_{\mathfrak{T}}^{*} y$.

(99) If $x, u \Rightarrow_{\mathfrak{T}}^{*} y$ and $y, v \Rightarrow_{\mathfrak{T}}^{*} z$, then $x, u^{\frown} v \Rightarrow_{\mathfrak{T}}^{*} z$.

(100) If \langle\rangle$_{E} \notin \operatorname{rng} \operatorname{dom}\left(\right.$ the transition of $\mathfrak{T}$ ), then $x,\langle\rangle_{E} \Rightarrow_{\mathfrak{T}}^{*} y$ iff $x=y$.

(101) If \langle\rangle$_{E} \notin \operatorname{rng} \operatorname{dom}$ (the transition of $\mathfrak{T}$ ), then if $x,\langle e\rangle \Rightarrow_{\mathfrak{T}}^{*} y$, then $x,\langle e\rangle \Rightarrow \mathfrak{T}$ $y,\langle\rangle_{E}$.

(102) If $\mathfrak{T}$ is deterministic, then if $x_{1}, x_{2} \Rightarrow_{\mathfrak{T}}^{*} y_{1}$ and $x_{1}, x_{2} \Rightarrow_{\mathfrak{T}}^{*} y_{2}$, then $y_{1}=y_{2}$.

\section{Reachable States}

Let us consider $E, F, \mathfrak{T}, x, X$. The functor $x$-succ $\mathfrak{T}(X)$ yields a subset of $\mathfrak{T}$ and is defined as follows:

(Def. 8) $x$ - $\operatorname{succ}_{\mathfrak{T}}(X)=\left\{s: \bigvee_{t}\left(t \in X \wedge t, x \Rightarrow_{\mathfrak{T}}^{*} s\right)\right\}$.

The following propositions are true:

(103) $s \in x-\operatorname{succ}_{\mathfrak{T}}(X)$ iff there exists $t$ such that $t \in X$ and $t, x \Rightarrow_{\mathfrak{T}}^{*} s$.

(104) If \langle\rangle$_{E} \notin \operatorname{rng} \operatorname{dom}\left(\right.$ the transition of $\mathfrak{T}$ ), then \langle\rangle$_{E}$-succ $\mathfrak{T}(S)=S$.

(105) Let $\mathfrak{T}_{1}$ be a non empty transition-system over $F_{1}$ and $\mathfrak{T}_{2}$ be a non empty transition-system over $F_{2}$. Suppose the carrier of $\mathfrak{T}_{1}=$ the carrier of $\mathfrak{T}_{2}$ and the transition of $\mathfrak{T}_{1}=$ the transition of $\mathfrak{T}_{2}$. Then $x$ - $\operatorname{succ}_{\mathfrak{T}_{1}}(X)=$ $x-\operatorname{succ}_{\mathfrak{T}_{2}}(X)$. 


\section{REFERENCES}

[1] Grzegorz Bancerek. Cardinal numbers. Formalized Mathematics, 1(2):377-382, 1990.

[2] Grzegorz Bancerek. The fundamental properties of natural numbers. Formalized Mathematics, 1(1):41-46, 1990.

[3] Grzegorz Bancerek. The ordinal numbers. Formalized Mathematics, 1(1):91-96, 1990.

[4] Grzegorz Bancerek. Reduction relations. Formalized Mathematics, 5(4):469-478, 1996.

[5] Grzegorz Bancerek and Krzysztof Hryniewiecki. Segments of natural numbers and finite sequences. Formalized Mathematics, 1(1):107-114, 1990.

[6] Czesław Byliński. Functions and their basic properties. Formalized Mathematics, 1(1):5565, 1990.

[7] Czesław Byliński. Functions from a set to a set. Formalized Mathematics, 1(1):153-164, 1990.

[8] Czesław Byliński. Some basic properties of sets. Formalized Mathematics, 1(1):47-53, 1990.

[9] Agata Darmochwał. Finite sets. Formalized Mathematics, 1(1):165-167, 1990.

[10] Karol Pąk. The Catalan numbers. Part II. Formalized Mathematics, 14(4):153-159, 2006, doi:10.2478/v10037-006-0019-7.

[11] Andrzej Trybulec. Domains and their Cartesian products. Formalized Mathematics, 1(1):115-122, 1990.

[12] Andrzej Trybulec. Tuples, projections and Cartesian products. Formalized Mathematics, 1(1):97-105, 1990.

[13] Michał Trybulec. Formal languages - concatenation and closure. Formalized Mathematics, 15(1):11-15, 2007, doi:10.2478/v10037-007-0002-y.

[14] Zinaida Trybulec. Properties of subsets. Formalized Mathematics, 1(1):67-71, 1990.

[15] Tetsuya Tsunetou, Grzegorz Bancerek, and Yatsuka Nakamura. Zero-based finite sequences. Formalized Mathematics, 9(4):825-829, 2001.

[16] Edmund Woronowicz. Relations and their basic properties. Formalized Mathematics, 1(1):73-83, 1990.

[17] Edmund Woronowicz. Relations defined on sets. Formalized Mathematics, 1(1):181-186, 1990.

Received May 5, 2009 